\title{
Stretchable and durable inverse vulcanized polymers with chemical and thermal recycling
}

\author{
Peiyao Yan*,1 Wei Zhao, ${ }^{1}$ Samuel J. Tonkin, ${ }^{2}$ Justin M. Chalker, ${ }^{2}$ Tara L. Schiller ${ }^{3}$ and Tom Hasell*1 \\ ${ }^{1}$ Department of Chemistry, University of Liverpool, Crown Street, Liverpool L69 7ZD (UK); ${ }^{2}$ Institute for Nanoscale \\ Science and Technology, College of Science and Engineering, Flinders University Bedford Park, South Australia 5042, \\ Australia; ${ }^{3}$ WMG, University of Warwick, Coventry CV4 7AL, UK. \\ KEYWORDS: inverse vulcanization, crosslinked sulfur polymer, chemical recycling and thermal recycling.
}

\begin{abstract}
Inverse vulcanized polymer materials have received considerable attention as a way to use sulfur, an industrial by-product, as starting material for synthesis. The resulting high-sulfur content polymers have also been investigated because their properties give rise to promising applications like infrared imaging, energy storage, and heavy metal capture due to their unique structure. However, synthesis of a flexible sulfur polymer network which shows good mechanical properties combining high strength, high elongation, and high toughness is still a significant challenge. Moreover, further exploration of the properties of sulfur polymers to better understand the relationship between the polymers' structure with their performance is still needed. Here, a range of crosslinked sulfur polymers with high tensile elongation and toughness, and without losing high strength were successfully synthesized. The obtained crosslinked sulfur polymers show high solvent tolerance in most organic solvents but are demonstrated to be chemically de-crosslinked in polar solvents dimethylformamide, dimethylacetamide and $\mathrm{N}$-methyl-2-pyrrolidone and can be re-crosslinked after removing the solvent due to the high sulfur ranks present in the polymer network. Despite the significantly improved mechanical properties, highly efficient thermal recycling performance typical of inverse vulcanized polymers was retained. Flexibility and durability, combined with chemical and thermal recycling, could open a new door for wider applications of inverse vulcanized polymers.
\end{abstract}

\section{INTRODUCTION}

Inverse vulcanization, coined by Jeffery Pyun et al., ${ }^{1}$ is a method of combining inorganic sulfur and organic monomers into a new type of sulfur-containing polymer. ${ }^{2,3}$ Sulfur, as an industrial by-product, is cheap and abundant.4, ${ }^{5}$ Inverse vulcanization makes it possible to transform this waste raw material into useful polymeric materials. The material obtained through this method is a kind of polymer network, based on sulfur chains crosslinked through organic co-monomers, which shows unique and interesting properties which most traditional polymers lack such as high refractive index 6 and intrinsic dynamic function ${ }^{7}$. To date, various promising applications of this kind of polymer, such as infrared imaging, ${ }^{8-13}$ Li-sulfur batteries, ${ }^{14-16}$ antibacterial materials, ${ }^{17}$ healable materials, ${ }^{13}$, ${ }^{18-20}$ fertilizers, $^{21}$ heavy metal capture, ${ }^{22-26}$ adhesives $^{27}$ or crosslinking agent, ${ }^{28-30}$ have been developed by varying crosslinker categories or adjusting stoichiometry of the comonomers. For example, crosslinkers 1,3diisopropylbenzene, ${ }^{8} \quad$ 1,3,5-tri-isopropenylbenzene,13 tetravinyltin,12 1,4-butanediol divinyl ether, and di (ethylene glycol) divinyl ether etc. ${ }^{10}$ were used in infrared optical applications; crosslinkers limonene, ${ }^{22}$ myrcene, ${ }^{24}$ dicyclopentadiene, ${ }^{23}$ and farnesene etc. ${ }^{5}$ were used in heavy metal capture. Concurrently, basic parameters of the polymers, for instance, glass transition temperature $\left(T_{g}\right)$, solubility, thermal properties and mechanical properties could be altered by changing crosslinker.
However, it is still hard to obtain inverse vulcanized polymers combining high strength, high elongation, and high toughness simultaneously, resulting from obvious contradiction between high bonding interactions for strength with molecular chains arrangement for stretchability, as well as the weakness of sulfur-sulfur (S-S) backbone bonds. Typically, inverse vulcanized polymers are formed by reacting sulfur with a crosslinker or blending crosslinkers in one step, so fully cured polymers generally have relatively high strength, high $T_{g}$, and high solvent tolerance, but very low breaking strain $(<15 \%)$ caused by their highly crosslinked structures. ${ }^{11}$, 13, 20, 31-37 Conversely, not fully crosslinked polymers can be formed as stretchable material with low $T_{g}$, but all have very low strength $(<2.0$ $\mathrm{MPa}$ ), and most of them are soluble in common solvents. ${ }^{11}$ $12,19,33,38-40$ This disadvantage of inverse vulcanized polymers limits their wider application in the areas that require the materials to have multiple tolerance to complex environments. Hence, it's highly desirable to identify flexible crosslinked sulfur polymers with high strength, high strain, high toughness and great solvent tolerance to allow wider potential applications in practical environments. Moreover, as inverse vulcanized polymers have a short developing history, there is still much needed to be done to discover, understand and explore the relationship between polymer structure and performance. Recently, the Chalker and Hasell laboratories discovered that pyridine and other nucleophilic amines can catalyze S- 


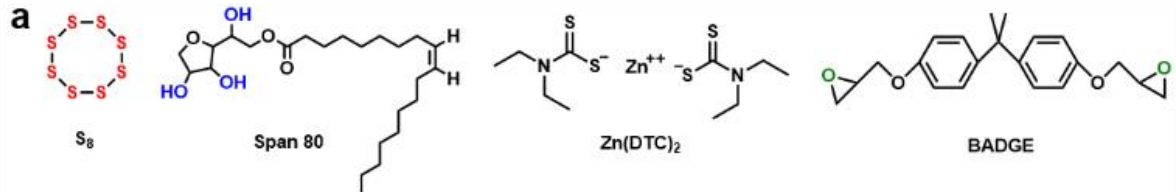

b Synthetic Step 1:

Synthetic Step 2:

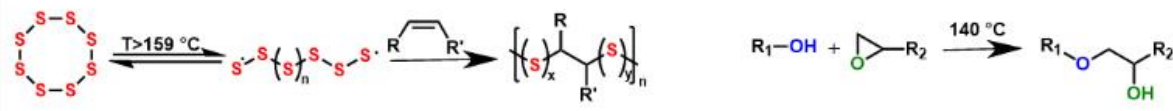

C Polymer network:

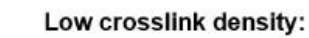

High crosslink density:
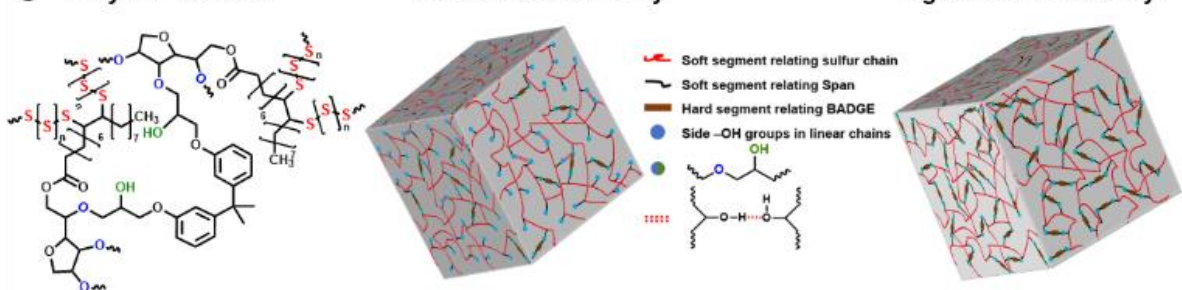

Figure 1. Schematic for the designed inverse vulcanized polymers: a) monomer structures, b) synthesis process, and c) polymer structure and network architecture illustration.

S metathesis in polysulfide polymers, which is a unique and useful chemical behavior of inverse vulcanized polymers warranting further investigation. ${ }^{41}$ They showed this reaction can be used to repair and recycle polymers made by inverse vulcanization. In our study here, we demonstrated for the first time that the solvents dimethylformamide (DMF), dimethylacetamide (DMAc) and N-methyl-2-pyrrolidone (NMP) facilitate homolytic cleavage of S-S bonds of sulfur polymers when sulfur rank is 3 or higher. This surprising discovery was demonstrated to be useful in de-crosslinking, recycling and reforming of these polysulfide polymers. Even for traditional thermoset materials, selective solvent degradation and recycling are difficult to achieve.

Regarding the problems raised above, we provide a potential solution starting from the design of a polymer structure using two-step inverse vulcanization as reported in our previous work. ${ }^{33}$ We propose that a low-activity crosslinker, which will react with the functional groups of the prepolymer, can allow the linear part of the polymer more time for chain growth in order for the molecular weight of those segments to increase. Here, the crosslinker acts as not only as a crosslinking agent but also a solvent to reduce the viscosity of reaction system, which makes the crosslinking reaction more uniform, forming a more homogeneous copolymer. Theoretically, higher molecular weight of the linear segments should endow higher ductility, while rigid crosslinking connections should provide high strength. To combine those two considerations in this work, span 80 (Span), a trifunctional aliphatic monomer containing a carbon-carbon double bond, was used to react with sulfur to form a linear prepolymer first, and difunctional aromatic monomer bisphenol A diglycidyl ether (BADGE) was selected as crosslinker in the second synthesis step following the principle that hydroxyl groups (on the Span) have a low reactivity with epoxy groups (on the BADGE) to form crosslinked sulfur polymers. The produced polymers show high elongation as designed, as well as high strength and toughness. A range of combinations of strength, strain, young's modulus, toughness, $T_{g}$, solubility and hardness were altered by adjusting the stoichiometric ratio of functional monomers. The resultant materials with crosslinked structure have a significant improvement in mechanical properties compared with the reported sulfurbased polymers, which allow them to resist deformations by stretching, bending or twisting at same time, resulting in a reshaping and shape memory property that allows complex 3D shape deformation and recovery which could not be achieved if the polymer has poor mechanical properties or a low degree of crosslinking. Owing to the widely known dynamic property of the intrinsic S-S bonds of polymer networks, the polymers show a good thermallyinduced recycling, healing, and reprogramming abilities via network rearrangement, but show an impressive higher recovery efficiency combined with improved mechanical properties in comparison to other reported sulfur-based polymer networks. More importantly, it is demonstrated for the first time here that DMF, DMAc and NMP are able to facilitate S-S bond cleavage, which enabled the polymer network to break and reconnect dynamically.

\section{RESULTS AND DISSCUSION}

\section{Design and synthesis of inverse vulcanized polymers Introducing a new cross linkage bond into sulfur}

polymers through inverse vulcanization in two steps. A trifunctional monomer, Span containing a carbon-carbon double bond was used here to react with sulfur $\left(\mathrm{S}_{8}\right)$ to form a stable linear polymer in the presence of catalyst zinc diethyldithiocarbamate (Zn (DTC) 2 ), which has been demonstrated in our previous work ${ }^{33}$, and BADGE was considered as secondary crosslinker, due to the reasons described in the introduction section, to crosslink the linear parts into polymer networks. Chemical structures of those monomers are shown in Figure. 1a. To realize this 

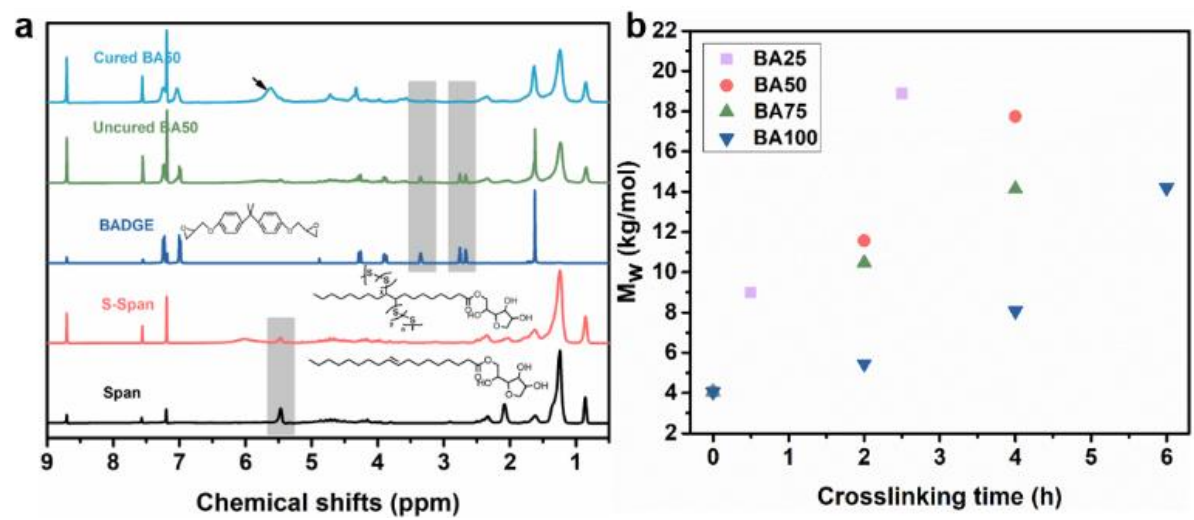

Figure. 2 Monitoring the polymer synthesis using 1H NMR and GPC in THF: a) ${ }^{1} \mathrm{H}$ NMR spectra of raw materials and obtained polymer BA50 and b) weight average molecular weight of uncured polymers as a function of crosslinking time.

experimental idea, a control experiment for the reaction of Span with BADGE was carried out to evaluate the possibility. The schematic of this reaction is shown in Figure. S1. Nuclear magnetic resonance spectroscopy (NMR) and Fourier transform infrared spectroscopy (FTIR) were used to monitor the reaction. Comparing the integral ratio of typical peaks from ${ }^{1} \mathrm{H}$ NMR spectra of monomers BADGE and Span (Figure. S2 and Figure. S4) with that from the ${ }^{1} \mathrm{H}$ NMR spectrum of the product (Figure. S3 and Figure. S5), we can see that peaks at " $\delta \approx " 3.31$ ppm, 2.86 ppm and 2.72 ppm which belongs to the epoxy group from BADGE decreased as the reaction proceeds, and a peak at " $\delta \approx " 5.34$ ppm belonging to the $\mathrm{C}=\mathrm{C}$ bond from Span had no change after the reaction. Also, from FT-IR spectra of monomers and the product in Figure. S6, it is obvious that the typical peak at $\sim 912 \mathrm{~cm}^{-1}$ of the epoxy group ${ }^{42}$ decreased after reaction. This result confirmed the epoxy groups in BADGE can react with hydroxyl groups in Span to form a crosslinked structure and there is no selfpolymerization of Span occurring during the heating process. Thus, inverse vulcanization of $S_{8}$, Span and BADGE formed sulfur polymer networks as shown in Figure. 1b. Through adjusting stoichiometric ratio between hydroxyl groups and epoxy groups, a wide range of crosslink densities of polymer networks were obtained as shown in Figure. 1c. Polymers were named as BA-x, where $x$ represents stoichiometric ratio value, and higher value means a higher degree of crosslinking in the polymer. Detailed illustration and experimental procedures about polymer design and synthesis can be found in the Supporting Information (SI). It is worth noting that toxic $\mathrm{H}_{2} \mathrm{~S}$ gas may be generated during inverse vulcanization, requiring operator awareness for safety.

Characterizations of obtained polymers. NMR was performed to monitor the formation of the expected polymers' structure. Due to our polymer being insoluble in most common solvents, discussed in detail in the next section, it is difficult to get a clear NMR spectrum of the polymer using solution NMR in common solvents. However, as mentioned above, pyridine recently was found to be able to chemically break S-S bonds allowing inverse vulcanized networks to dissolve. ${ }^{41}$ There should be little signal change of chemical shifts belonging to hydrogen or carbon in the polymer from breaking the S-S bonds in this way. So, deuterated pyridine was successfully used as a solvent for
NMR characterization here. The monitoring method is based on the integral ratio change of a reactive bond with an unreactive bond, which is explained detailed in SI (Figures. S7-S15). Taking polymer BA50 as an example to analyze ${ }^{1} \mathrm{H}$ NMR results, as shown in Figure. $2 \mathrm{a}$, it can be seen that the peak (" $\delta \approx " 5.47 \mathrm{ppm}$ ) belonging to hydrogens of the $\mathrm{C}=\mathrm{C}$ bond, and the peaks (" $\delta \approx " 3.35 \mathrm{ppm}$ and $" \delta \approx "$ $2.67,2.76 \mathrm{ppm}$ ) attributed to hydrogens in the epoxy group all disappeared after reaction. In addition, there is a new peak (" $\delta \approx " 5.63 \mathrm{ppm}$ ) formed which belongs to hydrogens in formed $-\mathrm{OH}$ groups after reaction. Moreover, ${ }^{13} \mathrm{C} \mathrm{NMR}$ spectra of raw materials and obtained product also can been found in Figures. S13-S16, which further prove all starting materials were consumed and new linkage bonds were formed as expected. Additionally, monitoring of polymer BA100 formation using ${ }^{1} \mathrm{H}$ NMR and ${ }^{13} \mathrm{C}$ NMR spectra was conducted, and results are shown in Figure. S17 and Figure. S18, which suggests all monomers reacted as expected and a polymer network with a new kind of cross linkage bond was formed. Gel permeation chromatography (GPC) was used to monitor polymer chain growth during crosslinking reaction, thanks to the uncured polymers being soluble in common organic solvents. Figure. $2 \mathrm{~b}$ shows that there is a trend of increasing molecular weight with crosslinking reaction time for each polymer, suggesting the reaction happened as expected and polymers undertake a uniform polymer chain growth during the crosslinking reaction. The typical GPC curves of each polymer are shown in Figure. S19, where specific molecular weight data and dispersity of each polymer in specific reaction time can be found.

FT-IR, Differential scanning calorimetry (DSC), thermogravimetric analysis (TGA) and X-ray diffraction (XRD) were used to characterize the structure and thermal properties of obtained polymers. According to the FT-IR spectra of Span and pre-polymer S-Span (Figure. S20a), the peak attributed to stretching vibration of $\mathrm{C}=\mathrm{C}-\mathrm{H}$ and $\mathrm{C}=\mathrm{C}$ in Span at $3080 \mathrm{~cm}^{-1}$ and $1600 \mathrm{~cm}^{-1}$ disappeared after reacting with sulfur, and a new peak belonging to C-S bond at 465 $\mathrm{cm}^{-1}$ was formed. Additionally, from FT-IR curves of BADGE and polymer BA100, the typical peak belonging to deformation of epoxy ring in monomer BADGE at $912 \mathrm{~cm}^{-1}$ totally disappeared after polymerization and there is no change for other typical peaks belonging to Span and BADGE. This indicates that the $\mathrm{C}=\mathrm{C}$ bonds were consumed 

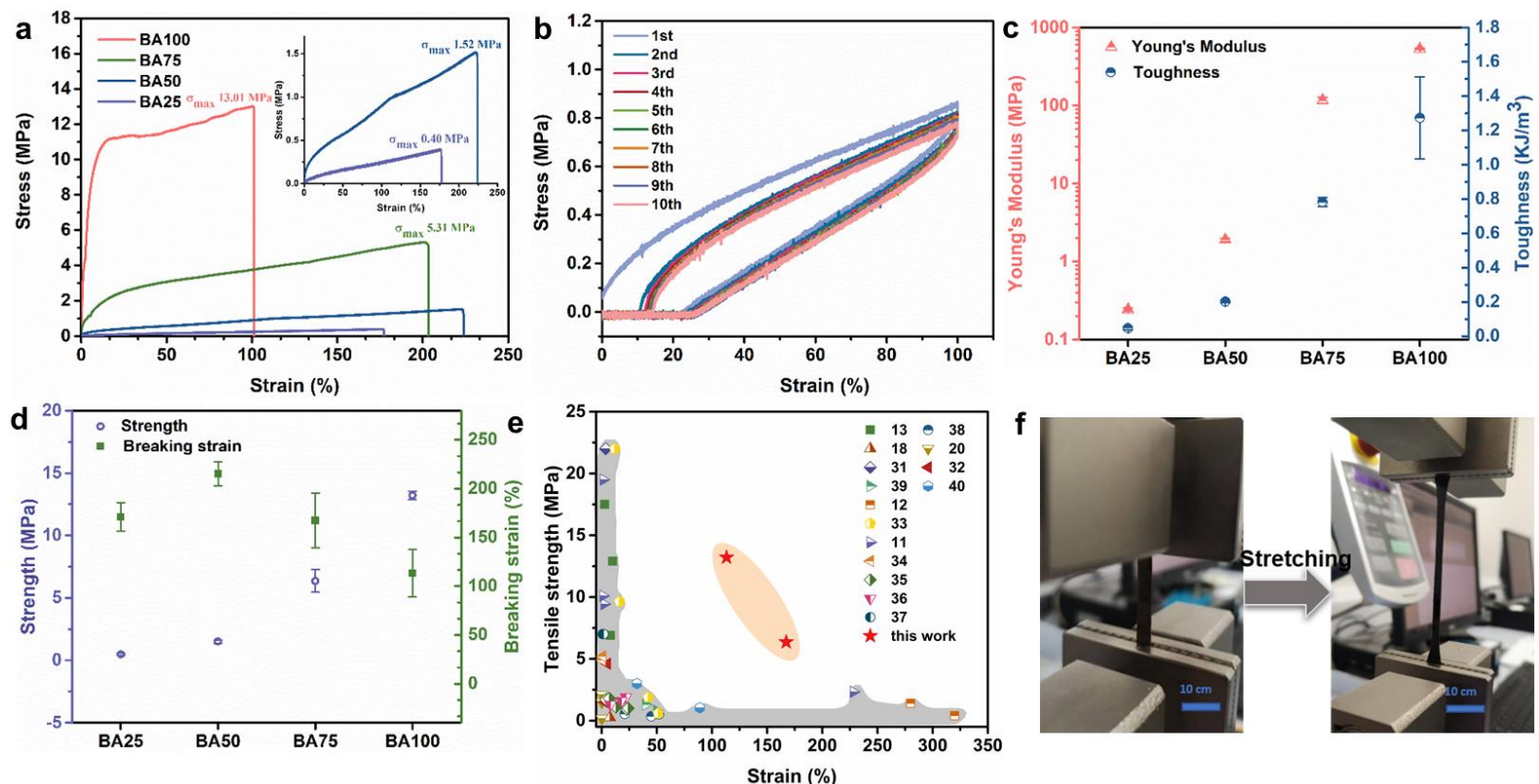

Figure. 3 a) The typical stress-strain curves of obtained polymers under strain rate $10 \mathrm{~mm} / \mathrm{min}$. b) Stress-strain curves in cyclic tensile tests of polymer BA50 under strain rate $5 \mathrm{~mm} / \mathrm{min} . \mathrm{c}$ ), d) Average young's modulus, toughness, maximum strength and breaking strain of obtained polymers. e) Comparison of tensile strength and strain of our polymers with other sulfur-sulfur bond based inverse vulcanized polymers. f) photo record of polymer BA75 in stretching process.

by sulfur and epoxy groups were consumed by pre-polymer as expected. Moreover, the peak belonging to - $\mathrm{OH}$ groups of the polymer network shifted right compared with that of pre-polymer, indicating $\mathrm{H}$-bonds were formed inside the polymer network. Similar results for the other three polymer systems were also recorded (Figure. S20b), suggesting that all polymer networks with designed structure were obtained. DSC (Figure. S21) and XRD (Figure. S22) show that there is no unreacted crystalline sulfur remaining in the obtained polymers, and there is a significant crosslink density dependence of $T_{g}$ (polymers with higher designed crosslink density show higher $T_{g}$ ). TGA results (Figure. S23) reveal that all polymer networks have a good thermal stability, and the specific degradation temperature of each polymer can be found in Table S2.

Basic physical properties characterizations. Solubility experiments were performed to demonstrate the formation of a crosslinked structure in the obtained polymers. A detailed description of the experimental method can be found in SI, as well as photographic records (Figure. S24 and Figure. S25). From the results, we can see that all four polymers are insoluble in organic solvents such as toluene, tetrahydrofuran (THF), chloroform, acetone, and ethyl acetate (EA). Only a slight soluble fraction of some oligomers was dissolved by these solvents causing a light color change. There was no shape change in the polymers with $50 \%$ or higher crosslink density, even after soaking in toluene for ten days, although the least crosslinked sample, BA25, at only $25 \%$ crosslink density, did fragment. The gel fraction of each polymer in different solvents were calculated (Figure. S26). The polymers with higher theoretical crosslink degree have a higher gel fraction regardless of solvent type. In support of our expectation, the results of tensile tests (Figures. 3a, 3c and 3d, Table S2) suggest that all obtained polymers exhibit a highly stretchable performance (Figure. 3f) with high toughness and without losing high strength. Importantly, the mechanical properties of the polymers were exactly controlled by adjusting the crosslink density. Tensile strength, Young's modulus and toughness have a significant dependence on the crosslink density. In principle, rigid linkage bonds and intermolecular interactions can improve the strength and modulus of polymers but result in a high resistance to polymer chain rearrangement which is the main root of high elongation. On the contrary, lower degree of chemical connectivity can give high mobility and chain rearrangement activation to a polymer but low strength. For instance, polymer BA25 with the lowest crosslink density has the lowest strength $(0.48 \pm 0.07 \mathrm{MPa})$, Young's modulus $(0.24 \pm 0.01 \mathrm{MPa})$ and toughness $\left(47.12 \pm 8.97 \mathrm{~J} / \mathrm{m}^{3}\right)$ as less rigid linkage bonds cannot endow high strength and modulus to the polymer. Instead, polymer BA100 with the highest crosslink density has the highest strength $(13.21 \pm 0.34 \mathrm{MPa})$, Young's modulus $(470.65 \pm 11.64 \mathrm{MPa})$ and toughness $\left(1273.36 \pm 239.67 \mathrm{~J} / \mathrm{m}^{3}\right)$. The strength of other two polymers range in-between them. For elongation, polymer BA50 has the highest value $(215.15 \% \pm 12.23 \%)$ due to a suitable combination of rigid linkage with chain rearrangement activation. Notably, polymers were adjusted from typical elastic to typical plastic performance, as shown in Figure. S28, deformations of polymers BA25 and BA50 are recoverable but that of polymers BA75 and BA100 are permanent. Moreover, as shown in Figure. $3 \mathrm{~b}$, polymer BA50 has a good cyclic recoverable tensile property with the maximum strain $100 \%$ during its elastic deformation range, and there is only a small hysteresis in first cycle and no obvious stress decrease in following cycles (Figure. S29). As mentioned in the introduction, most reported inverse vulcanized polymers have poor mechanical properties; some of them can reach high strength but show very low stretch-ability, or high stretch-ability but have very low strength and low solvent tolerance. Compared with those 


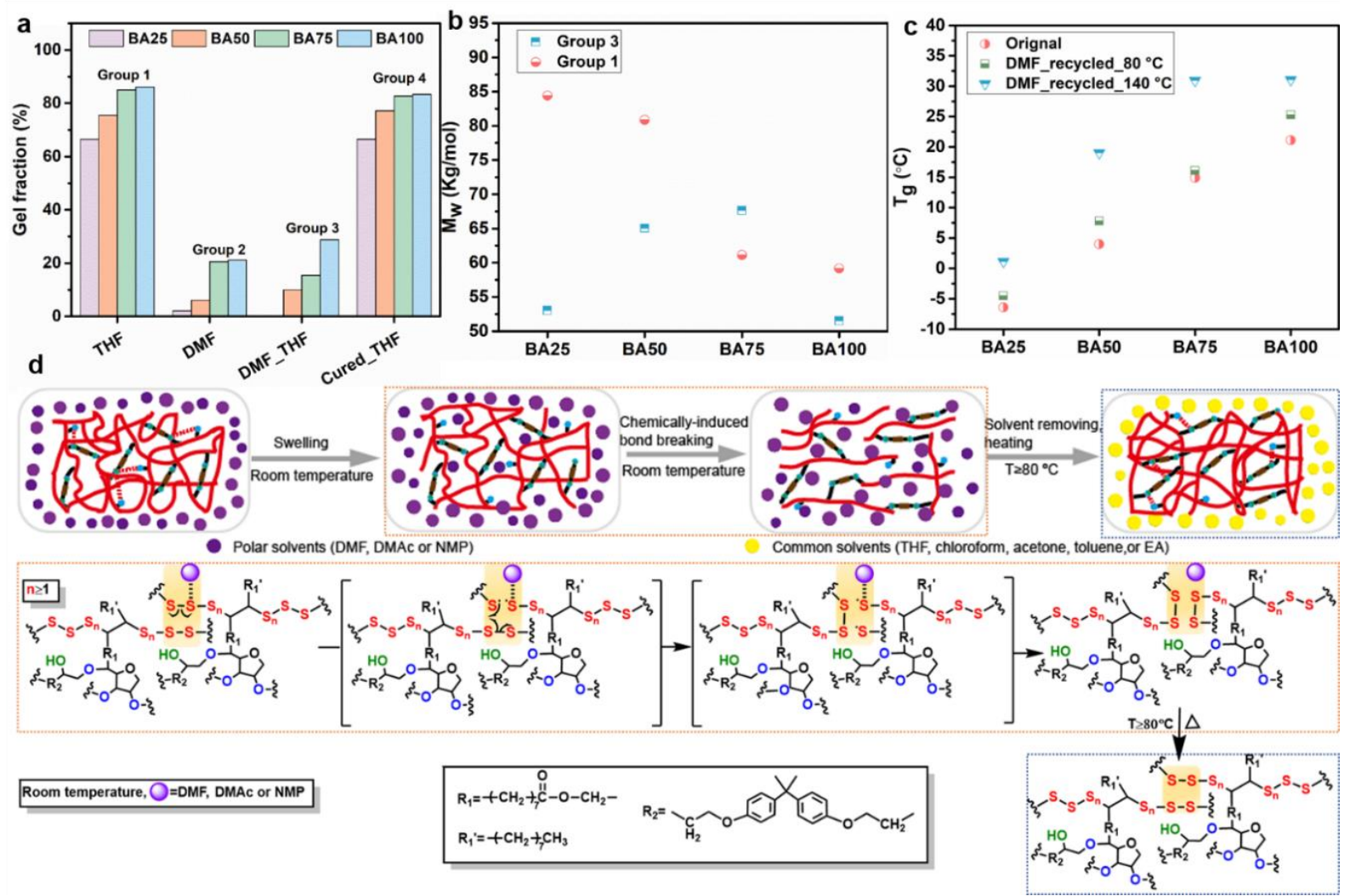

Figure. 4 a) Gel fraction of obtained polymers in THF, DMF, THF after DMF dissolved, and THF after DMF-induced recycled process. b) Comparison of the weight average molecular weight of the soluble fractions of each polymer in control group 1 and group 3 . c) DSC curves of polymers before and after DMF-induced recycled process under $80^{\circ} \mathrm{C}$ and $140{ }^{\circ} \mathrm{C}$ respectively. d) Proposed mechanism of chemically-induced recycling property (symbols for top of the figure can been found in figure 1c). The amide solvent is proposed to coordinate to the S-S bond, weakening it and lowering the energy needed for it to cleave to radicals.

reported polymers, our polymers fill the mechanical properties of the middle area of the combination which was lacking until now (Figure. 3e). Very recently, Jeffrey Pyun's research team reported a kind of segmented polyurethanes (PU) with sulfur with improved mechanical properties. ${ }^{43}$ While this is a notable advance, it differs significantly from the network structure of most inverse vulcanized polymer networks. Rather, the material they synthesized is based on aa branched PU-backbone sulfur containing polymer, rather than a crosslinked network and as a result is completely soluble.

\section{Functional properties and potential application of the crosslinked polymers}

Chemically-induced recycling ability. Various evidence shows that the obtained polymers have crosslinked structures, but those polymers were surprisingly found to be dissolved by DMF as well as pyridine. Pyridine is known to be able to cause chemical changes in inverse vulcanized polymers, rather than merely acting as a simple solvent. ${ }^{40}$ Therefore, in order to determine whether DMF is simply acting as a good solvent for polymers, indicating incomplete crosslinking, or if there is chemically-induced bond breaking happening, four control experiment groups were carried out (Figure. S30). This involved attempting to dissolve the polymers in either THF or DMF, or in THF after dissolving in DMF with or without a curing step in between. From the colour change of the four groups' results and calculated gel fraction value of each group (Figure. $4 \mathrm{a}$ ), it is obvious that polymers cannot be dissolved by THF (Group 1) but can be mostly dissolved by DMF (Group 2), and surprisingly the soluble fractions in DMF can be mostly dissolved by THF (Group 3). If DMF were acting simply as a solvent, the chemical structure would not be changed after dissolution, so the soluble fractions there should be still insoluble in THF. So, this result suggests that some chemical bond breaking happened during dissolution by DMF. The GPC analysis for soluble fractions of control Group 1 and control Group 3 was carried out to demonstrate molecular weight $\left(\mathrm{M}_{\mathrm{w}}\right)$ of polymer change during dissolving by DMF. Taking BA25 in Fig. 4b as an example for analysis, the Mw of $100 \%$ soluble BA25 in Group 3 is much lower than the Mw of 35\% soluble BA25 in Group 1. Normally, the soluble fractions of crosslinked polymers belong to some oligomers with low $\mathrm{M}_{\mathrm{w}}$. Here that the $\mathrm{M}_{\mathrm{w}}$ of partly soluble fractions much higher than the $\mathrm{M}_{\mathrm{w}}$ of totally soluble fractions means that the molecular weight of polymer BA25 was reduced through bond-breaking induced by DMF. For the other three polymers, similar results were obtained. The typical GPC curves with exact $\mathrm{M}_{\mathrm{w}}$ and dispersity of the four polymers are shown in Figure. S31. In addition, GPC of soluble fractions of control Group 2 was conducted using another GPC with eluent DMF. Due to equipment difference, those results cannot be directly compared with the results from GPC (THF), but the results suggest there is not much molecular growth for polymers even after around $20 \mathrm{~h}$ curing compared with GPC results of uncured polymers using the same equipment (Figure. S32). That further 

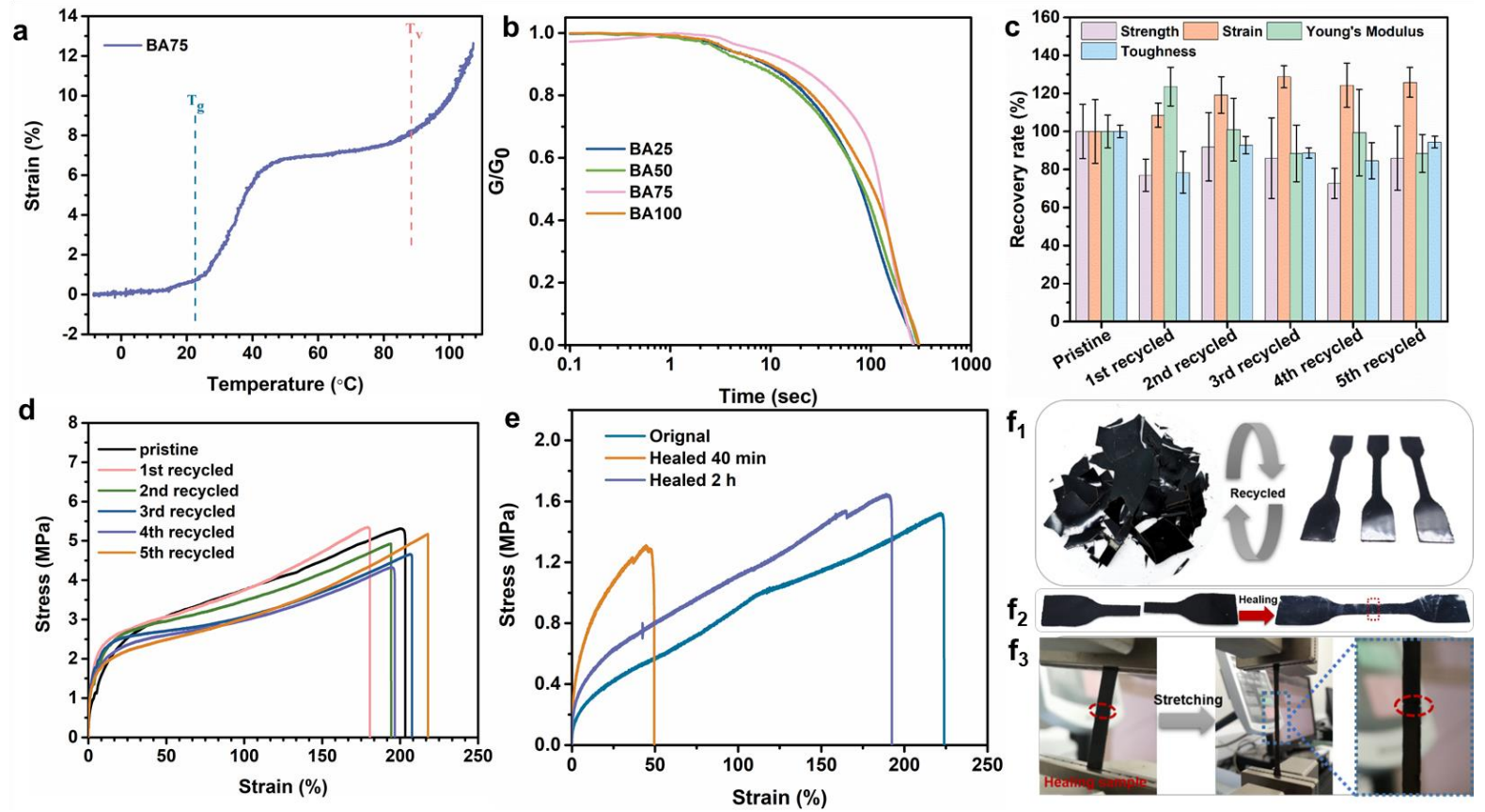

Figure. 5 a) Thermal expansion curves of polymer BA75. b) Stress relaxation curves of four polymers at $100^{\circ} \mathrm{C}$. c) Recovery rate of tensile strength, breaking strain, young's modulus and toughness of polymer BA75 after each cyclic reprocessed process. d) Typical stress-strain curves of recycled polymer BA75 in 5 recycle times. e) Typical stress-strain curves of polymer BA50 before and after self-healing for $40 \mathrm{~min}$ and $2 \mathrm{~h}$. f) Images of (f1) recycled process of polymer BA75, (f2) dog-bone self-healing sample of polymer BA50 and (f3) stretching process of self-healed polymer BA50.

indicates that DMF may induce chemical bond breaking as it shows little difference between the molecular weight of uncured and cured polymers, whereas early reaction stage products dissolved in THF did show an increase in Mw as a function of curing time (Figure. 2b). DMF can penetrate most polymers and, rather than acting merely as a solvent, it is also known to function as reagent, Lewis base, stabiliser, or catalyst in many reactions. ${ }^{44,} 45$ Model experiments on effect of DMF on polymers were carried out, GPC of two polymers S-Span and Span-BA in THF were conducted before and after dissolving in DMF. Figure. S33 shows there was a change in molecular weight distribution, which must require chemical changes, observed in the S-S based polymer S-Span but not in the polymer Span-BA which lacks disulfide bonds. Additionally, it was found that the obtained polymers can also be fully dissolved by NMP and DMAc (Figure. S34), which have similar chemical structure with DMF, suggesting that these polar solvents play a role in assisting with the cleavage of $\mathrm{S}-\mathrm{S}$ bonds. This theory was tested in a series of model crossover reactions. Accordingly, a mixture of dimethyl disulfide and dipropyl disulfide or dimethyl trisulfide and dipropyl trisulfide were exposed to DMF, DMAc, NMP, and THF (as a negative control), to determine if S-S metathesis and crossover was induced by any of these solvents (Figures. S35-S44). No exchange was observed for the disulfide system, in which the strength of the S-S bond is stronger. ${ }^{46}$ However, for the trisulfide model compounds, exchange was observed in all three amide containing solvents, with equilibrium being reached within 1 hour at room temperature. There was no such exchange in the absence of solvent, so the solvent is necessary for promoting the S-S metathesis. In THF some exchange did occur, but took much longer, with the crossover product only observed after 24 hours, and still not at equilibrium. To test if the observed trisulfide metathesis reactions occur by an anionic or radical mechanism, the same reactions were repeated but with 10 mol \% of (2,2,6,6-tetramethylpiperidin-1-yl)oxyl (TEMPO) in the reaction mixture. TEMPO is a common radical scavenger designed in this experiment to react with and quench any thiyl radicals formed in the S-S metathesis reaction. TEMPO would not inhibit the S-S metathesis if an ionic mechanism was operative. For all of the solvent systems, the crossover reaction was either entirely prevented, or severely inhibited, in the presence of TEMPO (Figures. S45-S54). These crossover experiments show that DMF, DMAc and NMP can promote an S-S metathesis reaction in trisulfides, but not disulfides, suggesting that a relatively high sulfur rank in the crosslinked polymers enables their dissolution. If the crosslinked polymers contained a significant proportion of disulfide bonds, then the network would likely still be held together, even if some higher rank S-S bonds were broken. The inhibition of the crossover reaction by TEMPO suggests that the $S-S$ metathesis reaction proceeds through a radical mechanism.

Fractions soluble in DMF were found to become insoluble in THF again after further curing (Group 4 in Figure. 4a). According to Figure. S55a, FT-IR curves of polymer BA75 and chemically recycled BA75 are totally overlapped without any new peak appeared or original peak disappeared, illustrating that the recycled sample is chemically unchanged to the original. The same results can be obtained for other three polymers from Figure. S55b. Furthermore, it was found that chemically broken-down polymers could be recovered to a crosslinked state at mild temperatures like $80{ }^{\circ} \mathrm{C}$ and not only at the higher temperature of $140{ }^{\circ} \mathrm{C}$. Recycled samples of polymers 

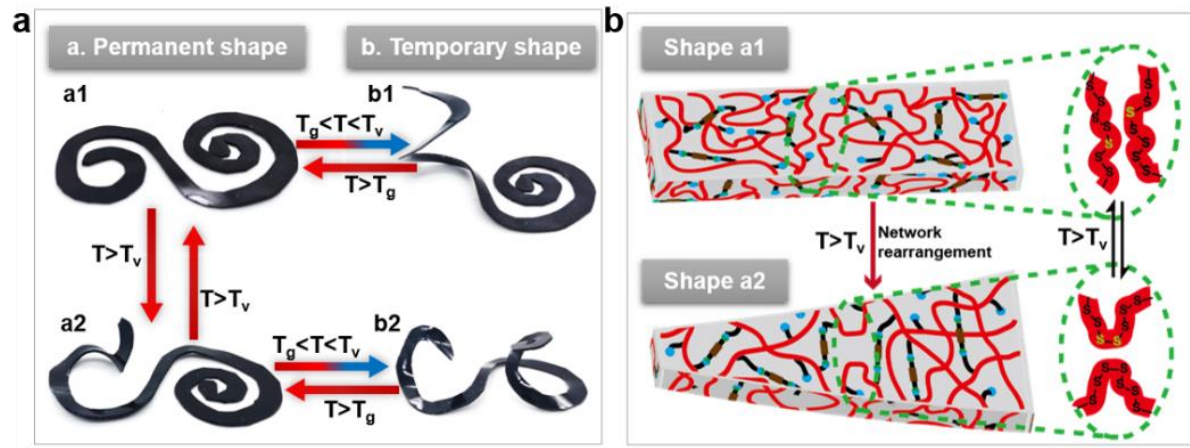

Figure. 6 a) Example of reversible shape memory and solid-solid reshaped performance of crosslinked polymer BA75. b) Mechanistic illustration of permanent reshaped property of the crosslinked sulfur polymer.

mostly recovered their crosslinked densities (Figure. S56), and their $T_{g}$ showed effective recovery - with the higher curing temperature resulting in a higher $T_{g}$ after recycling (Figures. 4c and S57, S58). The same results (Figure. S59) were observed for other two solvents NMP and DMAc taking highest crosslinking density polymer BA100 as an example. A proposed mechanism by which amide solvents degrade the crosslinking of the networks is shown in Figure. $4 \mathrm{~d}$. Here, the DMF and structurally similar amide-based solvents, may assist in breaking the hydrogen bonding in the network, as well as reducing the energy needed to cleave weaker, higher rank, S-S bonds. A re-curing process gives the degraded polymer a chance to recover its crosslinking degree by a thermally activated S-S metathesis reactions, resulting in the recovered insolubility of the recycled polymer in common solvents.

Thermally-induced recycling, self-healing, solid-solid reprogramming and shape memory. It's widely known that disulfide bonds are a kind of reversible covalent bond that has been used in vitrimers and the research of recyclable polymers. ${ }^{47-49}$ Here, our polymers contain a prevalence of S-S bonds due to sulfur backbone. It is plausible that thermally-activated bond breaking and bond formation could happen within our polymer networks, endowing those crosslinked polymers recycling ability despite their chemically covalent connectivity. Dynamic mechanical analysis (DMA) was used to evaluate the thermal dynamic properties of polymers BA-X. As shown in Figure. 5a, polymer BA75 has an obvious shape deformation above topology freezing temperature $\left(T_{v}\right)$ at around $90^{\circ} \mathrm{C}$ in this testing system, demonstrating this crosslinked polymer can be reshaped under external force at higher temperature in short time. To be clear, $T_{v}$ is a concept different from the physical parameter $T_{g}$, which is not a constant value and is a relevant value for certain experimentally observed time frames..$^{50}$ Here, around $90^{\circ} \mathrm{C}$ is considered as a reference $T_{v}$ for polymer BA75 and the following experiments were designed according to this temperature. In addition, polymer BA100 has a higher $T_{v}$ compared with polymer BA75 (Figure. S60), as higher degree of chemical connectivity results in a higher limitation for the reversible bond breaking-formation reaction. Attractively, all obtained polymers show a similar high stress relaxation speed at 100 ${ }^{\circ} \mathrm{C}$, as shown in Figure. 5b, where all stress of polymers relaxed to zero by $300 \mathrm{~s}$. That means the crosslinked polymers BA-x can obtain a fast recycling speed without needing high temperature or harsh conditions. Additionally, activation energy $(28.6 \pm 8.5 \mathrm{~kJ} / \mathrm{mol})$ of polymer BA100 for dynamic bonds reaction was calculated according to Figure. S61. According to these results, we can expect that the crosslinked polymers can be recycled or reshaped rapidly without a typical solid-liquid transition present for linear polymers.

That good mechanical properties of a crosslinked structure polymer can be efficiently recycled many times, provides a highly promising property of the material. A crosslinked polymer that shows a poor mechanical property and has recycling ability, while interesting as proof of principle, is less relevant for many wider practical applications. Hence, reprocessing, self-healing, and reshaping experiments were performed on the polymers and tensile measurements were recorded for each recycled sample. The experimental procedure and photographic records can be found in Figure. 5f, SI, and Figures. S62 and S63. As shown in Figures. 5c and 5d, polymer BA75, which was reprocessed 5 times, presents a good recycling ability with average $82.7 \%$ strength recovery rate, $121.3 \%$ strain recovery rate, $100.2 \%$ Young's modulus recovery rate, and $87.8 \%$ toughness recovery rate during every cyclical experiment. The results of FT-IR, DSC and XRD for recycled samples in Figures. S65, S66 and S67 demonstrate that polymers' chemical structure remained unchanged, there was a slight increase of $T_{g}$, and there is no crystalline free sulfur formed during the reprocessing. Recycling experiments of the other three polymers were also carried out, and the results as shown in Figures. S68-S71 exhibit that all those crosslinked polymers show a good recovery rate of mechanical properties, where the strength or breaking strain of some of them can be fully recovered. According to Figures. S72-S75, the FT-IR, DSC, and XRD curves of recycled polymers BA-X show that all polymers are chemically stable and can be recycled without structure change or significant $T_{g}$ change. In addition, a self-healing experiment was carried out for polymer BA50, as shown in Figures. 5f1 and 5f3, the healed sample maintains its stretchable property thanks to re-connectivity of chemical linkage via dynamic reaction of S-S bond. According to the tensile test results in Figure 5e, the broken sample just can get about $25 \%$ recovery rate after healing for $40 \mathrm{~min}$ but could reach near $90 \%$ recovery rate when the healing time was extended to $2 \mathrm{~h}$. 
As we discussed in the section above, the obtained polymers show enhanced mechanical properties with good deformation resistance ability or flexibility to stretching, bending, or twisting, and all present a clear phase transition performance after being heated above $T_{g}$, which gives them an attractive function, shape memory behavior. And it was proved that the polymers could be recycled or repaired, so they could be potentially reshaped in the solid state upon the principle that the polymer network architecture can be altered via reversible reaction of dynamic S-S bonds. Hence, combining those two features, the distinct shape memory and reshaping property of polymer BA75 was investigated. Although we have shown the shape memory ability of sulfur polymers in our previous work, the previous material has a rigid structure compared with this material such that it could only achieve some simple shape changes - without any twisting or stretching force applied. ${ }^{32}$ However, here although the polymer BA75 has a relatively high crosslink density, it is flexible rather than rigid, meaning complex shape deformation and robust temporary shape maintenance can be easily achieved. As shown in Figure. 6a, an original twin spiral shape a1 can be reshaped into temporary shape b1 which is recoverable depending on the temperature applied, and could also be reprogrammed into a new permanent shape a 2 which is unrecoverable but still maintains an elastic shape memory property as shown in a new temporary shape b2. Two videos showing those processes are available in the supporting information. Permanent shape a1 also can be 'recovered' through reshaping of shape $a 2$, which is a kind of chemical recycling form difference with elastic/physical recovery of form. The mechanism of this function as illustrated in Figure. 6b: the polymer network topology will be arranged via dynamic exchange reaction between S-S bonds, resulting in shape change but without losing crosslinked connectivity. This interesting combination function of the polymers can be applied in multiple repetitions or combinations to achieve a variety of complex original shapes based on the requirement of shape memory function in specific needs. A simple example can be found in Figure. S76.

Potential application as substrate in stretchable sensor. Recently, investigations on conductive polymer composites, which are made from attaching conductive fillers like gold, silver or liquid metal into polymer substrates, have drawn much research attention because of the potential of the obtained polymer composites to be applied in sensors or wearable materials. ${ }^{51-55}$ Compared with traditional polymers, using a sulfur-containing polymer as substrate allows for a more stable or more homogenous conductive polymer composite with stronger binding ability between contact surfaces, as there will be coordinate bonds formed between metal and sulfur. ${ }^{19,56,57}$ Hence, our polymer based on a sulfur backbone should show an obvious advantage here, where a uniform dispersion of metal filler in polymer will be formed as shown in the scheme in Figure. 7a, compared with traditional polymers. However, there is only report ${ }^{19}$ which investigated the conductivity sensitivity of inverse vulcanized polymers during deformation until now, mainly because their poor mechanical properties limit deeper exploration on this field of application. In that work, the authors showed that liquid metal was composited with a matrix of inverse vulcanized polymer poly (S-di- isopropenyl benzene) and the obtained material showed resistive sensitivity toward compressive force. However, the polymer they synthesized does not have any stretch ability (breaking strain $<10 \%$ ) and showed a very low tensile strength $(\sim 0.26 \mathrm{MPa})$. Inspired by the above research background, we explored a new potential application of inverse vulcanized polymers as stretchable sensors, as our polymers show an improved mechanical property combining high strength with high elongation. Here, fully taking advantage of the chemically-induced recycling, nanosilver particles (Ag) were introduced into polymer BA50 through sonication of DMF solution (Figure. S77). The energy dispersive spectroscopy (EDS) mapping images of composite Ag-BA50 surface shown in Figure. S78 suggests that Ag particles were uniformly dispersed in the polymer matrix. The polymer composite Ag-BA50 shows a strong resistance sensitivity towards stretching force as shown in Figure. $7 \mathrm{~b}$, hence, it is able to be used in a potential application like stretchable sensor materials. It is beneficial for these polymers to be applied in conductivity sensitive materials, as they have various basic functions like repaired, reshaped, recycled and shape memory behavior, which could be combined with the sensitivity to make multifunctional materials. We have not undertaken indepth investigation here, as the focus of this work is on a new kind of material design and synthesis but our future research will expand on these initial findings and we hope these proof-of-principle results will enable deeper and wider research.
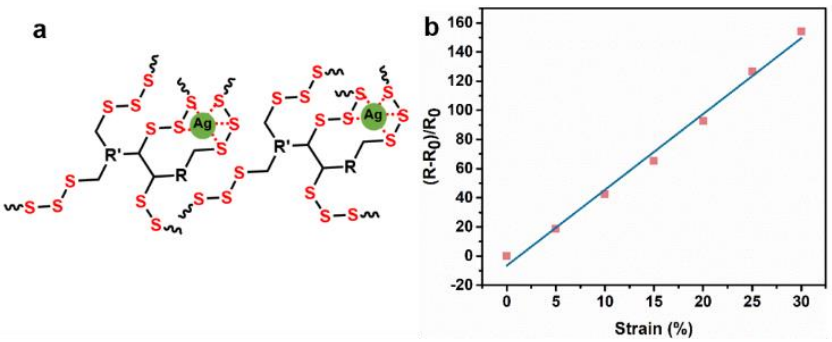

Figure. 7 a) Scheme of the interaction between silver with the polysulfide loops in sulfur-polymer. b) Electrical sensitivity of polymer composite Ag-BA50 under 0-30\% strain.

\section{CONCLUSIONS}

In summary, the synthesis of a series of flexible inverse vulcanized polymers with the combination of high strength, high elongation and high toughness was carried out. This was achieved by consideration of polymer structure design. The physical properties of these polymers, such as $T_{g}$, tensile strength, breaking strain, Young's modulus, toughness, and hardness, were proved to be altered by adjusting the stoichiometric ratio of the functional monomers, such as adjusting the breaking strain from $113.2 \%$ to $215.1 \%$. Importantly, the crosslinked polymers were demonstrated to be resistant to common solvents but could surprisingly be chemically dissolved and recycled in DMF, DMAc or NMP, which could allow intentional removal and recovery. Moreover, despite their crosslinked structure and improved mechanical properties, the polymers were found to still maintain highly recoverable thermal recycling. In addition, the polymers with rational crosslink density show excellent shape memory behavior with complex 
shape design, which would not be possible to achieve with poor mechanical properties. Finally, an example potential application as stretching sensors, enhanced by their unique sulfur-based structure and stretch ability, was illustrated.

\section{ASSOCIATED CONTENT}

This material is available free of charge via the Internet at http://pubs.acs.org.

Additional materials and methods, additional characterizations (PDF). Reversible shape memory property (MP4).

\section{AUTHOR INFORMATION}

\section{Corresponding Author}

* Tom Hasell: t0m@liverpool.ac.uk

* Peiyao Yan: Peiyao.Yan@liverpool.ac.uk

\section{Author Contributions}

P.Y. designed, synthesized, and characterized the materials. W.Z. performed XRD measurement. T.S. and P.H. performed DMA testing and assisted with interpretation. T.H. directed the project. S.J.T. performed the crossover model reactions. Data analysis and manuscript preparation were completed by P.Y., J.M.C., and T.H..

\section{ACKNOWLEDGMENT}

P.Y. thanks the China Scholarship Council (CSC) for awarding her a scholarship. T.H. holds a Royal Society University Research Fellowship. We thank Paul Hadlum for assistance with DMA measurements.

\section{REFERENCES}

(1) Chung, W. J.; Griebel, J. J.; Kim, E. T.; Yoon, H.; Simmonds, A. G.; Ji, H. J.; Dirlam, P. T.; Glass, R. S.; Wie, J. J.; Nguyen, N. A.; Guralnick, B. W.; Park, J.; Somogyi, A.; Theato, P.; Mackay, M. E.; Sung, Y. E.; Char, K.; Pyun, J., The use of elemental sulfur as an alternative feedstock for polymeric materials. Nat. Chem. 2013, 5, 518-524. (2) Lim, J.; Pyun, J.; Char, K., Recent approaches for the direct use of elemental sulfur in the synthesis and processing of advanced materials. Angew. Chem. Int. Ed. Engl. 2015, 54, 3249-3258.

(3) Zhang, Y.; Glass, R. S.; Char, K.; Pyun, J., Recent advances in the polymerization of elemental sulphur, inverse vulcanization and methods to obtain functional Chalcogenide Hybrid Inorganic/Organic Polymers (CHIPs). Polymer Chemistry 2019, $10,4078-4105$.

(4) Kutney, G., Sulfur: history, technology, applications \& industry. Chem.Tec. Publishing: 2007.

(5) Worthington, M. J. H.; Kucera, R. L.; Chalker, J. M., Green chemistry and polymers made from sulfur. Green Chemistry 2017, 19, 2748-2761.

(6) Kleine, T. S.; Glass, R. S.; Lichtenberger, D. L.; Mackay, M. E.; Char, K.; Norwood, R. A.; Pyun, J., 100th Anniversary of Macromolecular Science Viewpoint: High Refractive Index Polymers from Elemental Sulfur for Infrared Thermal Imaging and Optics. ACS Macro. Letters. 2020, 9, 245-259.

(7) Zhang, Y.; Pavlopoulos, N. G.; Kleine, T. S.; Karayilan, M.; Glass, R. S.; Char, K.; Pyun, J., Nucleophilic Activation of Elemental Sulfur for Inverse Vulcanization and Dynamic Covalent Polymerizations. Journal of Polymer Science Part A: Polymer Chemistry 2018, 57, 712.

(8) Griebel, J. J.; Namnabat, S.; Kim, E. T.; Himmelhuber, R.; Moronta, D. H.; Chung, W. J.; Simmonds, A. G.; Kim, K. J.; van der Laan, J.; Nguyen, N. A.; Dereniak, E. L.; Mackay, M. E.; Char, K.; Glass, R. S.; Norwood, R. A.; Pyun, J., New infrared transmitting material via inverse vulcanization of elemental sulfur to prepare high refractive index polymers. Adv. Mater. 2014, 26, 3014-3018.

(9) Boyd, D. A.; Nguyen, V. Q.; McClain, C. C.; Kung, F. H.; Baker, C. C.; Myers, J. D.; Hunt, M. P.; Kim, W.; Sanghera, J. S., Optical Properties of a Sulfur-Rich Organically Modified Chalcogenide Polymer Synthesized via Inverse Vulcanization and Containing an Organometallic Comonomer. ACS Macro. Letters. 2019, 8, 113-116. (10) Jang, W.; Choi, K.; Choi, J. S.; Pyun, J.; Lim, J.; Char, K.; Im, S. G., One-step vapor-phase synthesis of transparent high refractive index sulfur-containing polymers. Science advances 2020, 6, eabb5320.

(11) Kleine, T. S.; Nguyen, N. A.; Anderson, L. E.; Namnabat, S.; LaVilla, E. A.; Showghi, S. A.; Dirlam, P. T.; Arrington, C. B.; Manchester, M. S.; Schwiegerling, J., High refractive index copolymers with improved thermomechanical properties via the inverse vulcanization of sulfur and 1, 3, 5-triisopropenylbenzene. ACS Macro. Letters. 2016, 5, 1152-1156.

(12) Kuwabara, J.; Oi, K.; Watanabe, M. M.; Fukuda, T.; Kanbara, T., Algae-Inspired, Sulfur-Based Polymer with Infrared Transmission and Elastic Function. ACS Applied Polymer Materials 2020, 2, 5173-5178.

(13) Griebel, J. J.; Nguyen, N. A.; Namnabat, S.; Anderson, L. E.; Glass, R. S.; Norwood, R. A.; Mackay, M. E.; Char, K.; Pyun, J., Dynamic Covalent Polymers via Inverse Vulcanization of Elemental Sulfur for Healable Infrared Optical Materials. ACS Macro. Letters. 2015, 4, 862-866.

(14) Arslan, M.; Kiskan, B.; Cengiz, E. C.; Demir-Cakan, R.; Yagci, Y., Inverse vulcanization of bismaleimide and divinylbenzene by elemental sulfur for lithium sulfur batteries. European Polymer Journal 2016, 80, 70-77.

(15) Kang, H.; Kim, H.; Park, M. J., Sulfur-Rich Polymers with Functional Linkers for High-Capacity and Fast-Charging LithiumSulfur Batteries. Advanced Energy Materials 2018, 8.

(16) Bayram, O.; Kiskan, B.; Demir, E.; Demir-Cakan, R.; Yagci, Y., Advanced Thermosets from Sulfur and Renewable Benzoxazine and Ionones via Inverse Vulcanization. ACS Sustainable Chemistry \& Engineering 2020, 8, 9145-9155.

(17) Deng, Z.; Hoefling, A.; Théato, P.; Lienkamp, K., Surface Properties and Antimicrobial Activity of Poly(sulfur-co

-1,3-diisopropenylbenzene) Copolymers. Macromolecular Chemistry and Physics 2018, 219.

(18) Lin, H.-K.; Lai, Y.-S.; Liu, Y.-L., Cross-Linkable and Self-Foaming Polysulfide Materials for Repairable and Mercury Capture Applications. ACS Sustainable Chemistry \& Engineering 2019, 7, 4515-4522.

(19) Xin, Y.; Peng, H.; Xu, J.; Zhang, J., Ultrauniform Embedded Liquid Metal in Sulfur Polymers for Recyclable, Conductive, and Self-Healable Materials. Adv. Funct. Mater. 2019, 29.

(20) Karunarathna, M. S.; Lauer, M. K.; Thiounn, T.; Smith, R. C.; Tennyson, A. G., Valorisation of waste to yield recyclable composites of elemental sulfur and lignin. Journal of Materials Chemistry A 2019, 7, 15683-15690.

(21) Mann, M.; Kruger, J. E.; Andari, F.; McErlean, J.; Gascooke, J. R.; Smith, J. A.; Worthington, M. J. H.; McKinley, C. C. C.; Campbell, J. A.; Lewis, D. A.; Hasell, T.; Perkins, M. V.; Chalker, J. M., Sulfur polymer composites as controlled-release fertilisers. Org. Biomol. Chem. 2019, 17, 1929-1936.

(22) Crockett, M. P.; Evans, A. M.; Worthington, M. J.; Albuquerque, I. S.; Slattery, A. D.; Gibson, C. T.; Campbell, J. A.; Lewis, D. A.; Bernardes, G. J.; Chalker, J. M., Sulfur-Limonene Polysulfide: A Material Synthesized Entirely from Industrial By-Products and Its Use in Removing Toxic Metals from Water and Soil. Angew. Chem. Int. Ed. Engl. 2016, 55, 1714-1718.

(23) Lee, J.-Sing M.; Parker, D. J.; Cooper, A. I.; Hasell, T., High surface area sulfur-doped microporous carbons from inverse vulcanised polymers. Journal of Materials Chemistry A 2017, 5, 18603-18609.

(24) Parker, D. J.; Jones, H. A.; Petcher, S.; Cervini, L.; Griffin, J. M.; Akhtar, R.; Hasell, T., Low cost and renewable sulfur-polymers by 
inverse vulcanisation, and their potential for mercury capture. Journal of Materials Chemistry A 2017, 5, 11682-11692.

(25) Petcher, S.; Parker, D. J.; Hasell, T., Macroporous sulfur polymers from a sodium chloride porogen - a low cost, versatile remediation material. Environmental Science: Water Research \& Technology 2019, 5, 2142-2149.

(26) Scheiger, J. M.; Direksilp, C.; Falkenstein, P.; Welle, A.; Koenig, M.; Heissler, S.; Matysik, J.; Levkin, P. A.; Theato, P., Inverse Vulcanization of Styrylethyltrimethoxysilane-Coated Surfaces, Particles, and Crosslinked Materials. Angew Chem Int Ed Engl 2020, 59, 18639-18645.

(27) Herrera, C.; Ysinga, K. J.; Jenkins, C. L., Polysulfides Synthesized from Renewable Garlic Components and Repurposed Sulfur Form Environmentally Friendly Adhesives. ACS Appl. Mater. Interfaces. 2019, 11, 35312-35318.

(28) Wang, D.; Tang, Z.; Liu, Y.; Guo, B., Crosslinking diene rubbers by using an inverse vulcanised co-polymer. Green Chemistry 2020, $22,7337-7342$.

(29) Lauer, M. K.; Tennyson, A. G.; Smith, R. C., Inverse vulcanization of octenyl succinate-modified corn starch as a route to biopolymer-sulfur composites. Materials Advances 2021, 2, 2391-2397.

(30) Sun, L.; Gao, S.; Gui, X.; Liu, L.; Xu, K.; Liu, H., Renewable sulfurand monoterpenes-derived polysulfides as functional crosslinker for epoxy thermosets. European Polymer Journal 2020, 123.

(31) Lin, H. K.; Liu, Y. L., Reactive Hybrid of Polyhedral Oligomeric Silsesquioxane (POSS) and Sulfur as a Building Block for SelfHealing Materials. Macromol. Rapid. Commun. 2017, 38.

(32) Smith, J. A.; Green, S. J.; Petcher, S.; Parker, D. J.; Zhang, B.; Worthington, M. J. H.; Wu, X.; Kelly, C. A.; Baker, T.; Gibson, C. T.; Campbell, J. A.; Lewis, D. A.; Jenkins, M. J.; Willcock, H.; Chalker, J. M.; Hasell, T., Crosslinker Copolymerization for Property Control in Inverse Vulcanization. Chemistry - A European Journal 2019, 25, 10433-10440.

(33) Yan, P.; Zhao, W.; Zhang, B.; Jiang, L.; Petcher, S.; Smith, J. A.; Parker, D. J.; Cooper, A. I.; Lei, J.; Hasell, T., Inverse Vulcanized Polymers with Shape Memory, Enhanced Mechanical Properties, and Vitrimer Behavior. Angew. Chem. Int. Ed. Engl. 2020, 59, 13371-13378.

(34) Lauer, M. K.; Tennyson, A. G.; Smith, R. C., Green Synthesis of Thermoplastic Composites from a Terpenoid-Cellulose Ester. ACS Applied Polymer Materials 2020, 2, 3761-3765.

(35) Smith, A. D.; McMillen, C. D.; Smith, R. C.; Tennyson, A. G., Copolymers by Inverse Vulcanization of Sulfur with Pure or Technical-Grade Unsaturated Fatty Acids. Journal of Polymer Science 2020, 58, 438-445.

(36) Smith, A. D.; Smith, R. C.; Tennyson, A. G., Polymer cements by copolymerization of waste sulfur, oleic acid, and pozzolan cements. Sustainable Chemistry and Pharmacy 2020, 16.

(37) Thiounn, T.; Tennyson, A. G.; Smith, R. C., Durable, acidresistant copolymers from industrial by-product sulfur and microbially-produced tyrosine. RSC Advances 2019, 9, 3146031465.

(38) Khawaja, S. Z.; Vijay Kumar, S.; Jena, K. K.; Alhassan, S. M., Flexible sulfur film from inverse vulcanization technique. Materials Letters 2017, 203, 58-61.

(39) Diez, S.; Hoefling, A.; Theato, P.; Pauer, W., Mechanical and Electrical Properties of Sulfur-Containing Polymeric Materials Prepared via Inverse Vulcanization. Polymers (Basel) 2017, 9.

(40) Thiounn, T.; Karunarathna, M. S.; Slann, L. M.; Lauer, M. K.; Smith, R. C., Sequential crosslinking for mechanical property development in high sulfur content composites. Journal of Polymer Science 2020, 58, 2943-2950.
(41) Tonkin, S. J.; Gibson, C. T.; Campbell, J. A.; Lewis, D. A.; Karton, A.; Hasell, T.; Chalker, J. M., Chemically induced repair, adhesion, and recycling of polymers made by inverse vulcanization. Chem Sci 2020, 11, 5537-5546.

(42) Donald L. Pavia, G. M. L., George S. Kriz, Introduction to spectroscopy.

(43) Kang, K. S.; Phan, A.; Olikagu, C.; Lee, T.; Loy, D. A.; Kwon, M.; Paik, H. J.; Hong, S. J.; Bang, J.; Parker, W. O., Jr.; Sciarra, M.; de Angelis, A. R.; Pyun, J., Segmented Polyurethanes and Thermoplastic Elastomers from Elemental Sulfur with Enhanced Thermomechanical Properties and Flame Retardancy. Angew Chem Int Ed Engl 2021, 60, 22900-22907.

(44) Patschinski, P.; Zhang, C.; Zipse, H., The Lewis Base-Catalyzed Silylation of Alcohols? A Mechanistic Analysis. The Journal of organic chemistry 2014, 79, 8348-8357.

(45) Heravi, M. M.; Ghavidel, M.; Mohammadkhani, L., Beyond a solvent: triple roles of dimethylformamide in organic chemistry. RSC advances 2018, 8, 27832-27862.

(46) Lundquist, N. A.; Tikoalu, A. D.; Worthington, M. J.; Shapter, R.; Tonkin, S. J.; Stojcevski, F.; Mann, M.; Gibson, C. T.; Gascooke, J. R.; Karton, A., Reactive Compression Molding Post-Inverse Vulcanization: A Method to Assemble, Recycle, and Repurpose Sulfur Polymers and Composites. Chemistry-A European Journal 2020, 26, 10035-10044.

(47) Ni, C.; Chen, D.; Zhang, Y.; Xie, T.; Zhao, Q., Autonomous Shapeshifting Hydrogels via Temporal Programming of Photoswitchable Dynamic Network. Chemistry of Materials 2021, 33, 2046-2053.

(48) Trejo-Machin, A.; Puchot, L.; Verge, P., A cardanol-based polybenzoxazine vitrimer: recycling, reshaping and reversible adhesion. Polymer Chemistry 2020, 11, 7026-7034.

(49) Lee, J. M.; Choi, Y. S.; Noh, G. Y.; Lee, W.; Yoo, Y.; Kim, D. G.; Yoon, H. G.; Kim, Y. S., Spatiotemporally Controlled Plasticity and Elasticity in 3D Multi-Shape Memory Structures Enabled by Elemental Sulfur-Derived Polysulfide Networks with Intrinsic NIR Responsiveness. Macromol Rapid Commun 2020, 41, e2000013.

(50) Guerre, M.; Taplan, C.; Winne, J. M.; Du Prez, F. E., Vitrimers: directing chemical reactivity to control material properties. Chemical Science 2020, 11, 4855-4870.

(51) Ling, Y.; An, T.; Yap, L. W.; Zhu, B.; Gong, S.; Cheng, W., Disruptive, Soft, Wearable Sensors. Adv Mater 2020, 32, e1904664. (52) Lee, J.; Kim, S.; Lee, J.; Yang, D.; Park, B. C.; Ryu, S.; Park, I., A stretchable strain sensor based on a metal nanoparticle thin film for human motion detection. Nanoscale 2014, 6, 11932-11939.

(53) Li, G.; Wu, X.; Lee, D. W., A galinstan-based inkjet printing system for highly stretchable electronics with self-healing capability. Lab Chip 2016, 16, 1366-1373.

(54) Gao, Q.; Li, H.; Zhang, J.; Xie, Z.; Zhang, J.; Wang, L., Microchannel Structural Design for a Room-Temperature Liquid Metal Based Super-stretchable Sensor. Sci Rep 2019, 9, 5908.

(55) Ling, Y.; Guo, K.; Zhu, B.; Prieto-Simon, B.; Voelcker, N. H.; Cheng, W., High-adhesion vertically aligned gold nanowire stretchable electrodes via a thin-layer soft nailing strategy. Nanoscale Horizons 2019, 4, 1380-1387.

(56) Tang, S. Y.; Qiao, R. R.; Yan, S.; Yuan, D.; Zhao, Q. B.; Yun, G. L.; Davis, T. P.; Li, W. H., Microfluidic Mass Production of Stabilized and Stealthy Liquid Metal Nanoparticles. Small 2018, 14.

(57) Ren, Z.; Jiang, X.; Liu, L.; Yin, C.; Wang, S.; Yang, X., Modification of high-sulfur polymer using a mixture porogen and its application as advanced adsorbents for $\mathrm{Au}(\mathrm{III})$ from wastewater. Journal of Molecular Liquids 2021, 328. 


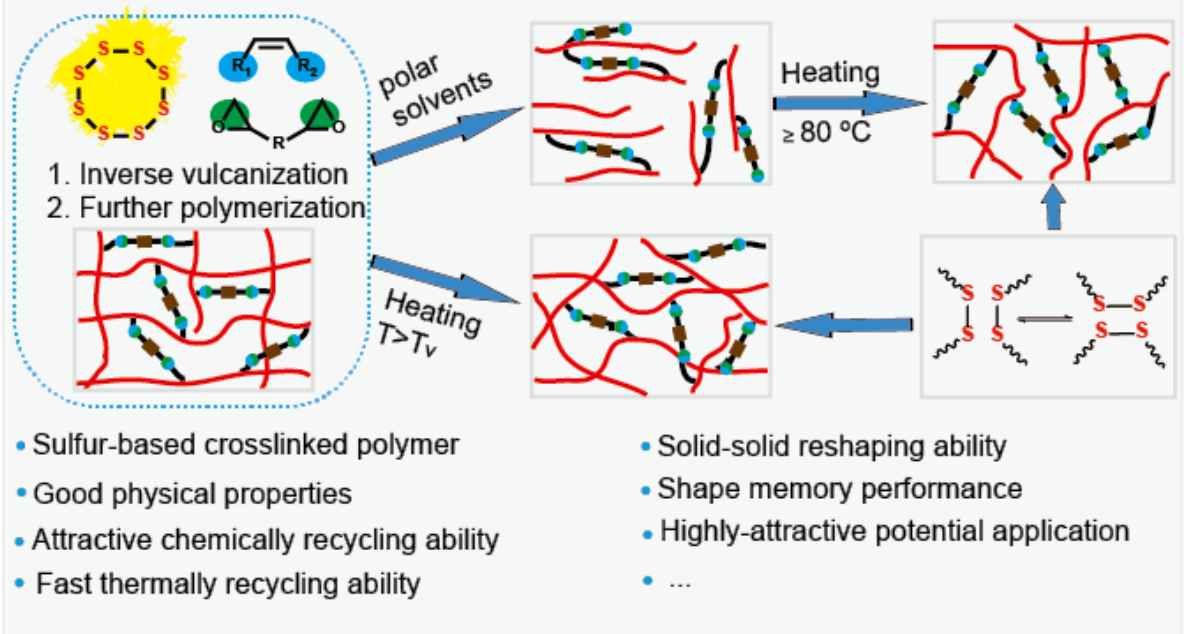

